\title{
Editorial
}

\section{Disaster Consequences and Collaboration}

\author{
Sanneke Kuipers
}

One century ago, right after the turn of the year, an up to 40 feet tall wave of sticky syrup flooded Boston's North End area with a speed of 35 miles an hour. There was no way to outrun the spill, that killed 21 and injured over 150. Once the 2.3 million gallons of molasses had burst from a storage tank in the Boston Harbor area, it formed a flood that destroyed everything in its path (McCann, 2016). A New York Times article on the fateful day reports on the course of events, the casualties and the damage, and the owners of the property involved. Two topics of particular interest stand out: the explosion and subsequent flood of molasses destroyed the adjacent fire station-ridding the area of its emergency services-so the first to come to the rescue were officers from a nearby nautical school, soon joined by police reserves and military from the nearest army station (NYT, January 15, 1919). Apparently unanticipated serendipity (La Porte, 2018, p. 260) and networked collaborative crisis governance (Kuipers and Welsh, 2017, p. 280) are of all times.

The New York Times of 1919 typically does not report on potential causes, responsibility or accountability issues, the public health effects, or the consequences for the directly affected citizens. For one thing, because it would take almost hundred years before a Harvard simulation based study would finally pinpoint causality (Shanahan, 2019). Most other crisis research interests such as accountability, health risks and citizens' perspectives would also require time to gain ground.

This first issue of Risk, Hazards and Crisis in Public Policy (RHCPP) of 2019 reports on disaster consequences for citizens, and on the intricacies of collaboration in crisis response. For starters, Jong and Dückers (2019) in their article, "The Perspective of the Affected" focus on psychosocial support in the aftermath of crisis. They conclude that their respondents highly valued fairness, equality, compassion and reliability in government long-term aid. Affected citizens also expected "government support in fulfilling event-related practical needs, and assumed that the government would use its capacity to align network partners 
and break down bureaucratic barriers" (2019, p. XX). Since explicit aftercare expectations by citizens confronted with disasters are an under-researched topic in the crisis and disaster literature, these empirical findings may provide valuable insights to practitioners. They confirm the expectation gaps outlined by Boin and 't Hart (2003, pp. 547-548), on the discrepancy between public expectations and what public leaders can actually deliver in crisis.

The next article, by Lai, Esnard, Wyczalkowski, Savage, and Shah (2019), examines another under-studied topic: the differing patterns of recovery after disaster. The authors look into risks and explanatory factors related to disaster recovery of schools. They find that low school attendance and a high number of economically disadvantaged students are significant risk factors for school recovery. The authors emphasize that their findings show once more that disasters do not affect citizens or communities equally, and that discrimination in impact persists in the long run. Policy initiatives aimed at recovery thus need to take into account that "relatively low performing schools are at highest risk for adverse outcomes post- disaster" (Lai et al., 2019, p. xx).

Post-disaster cooperation in recovery efforts between public institutions proves no easy task. Fisk, Good, and Nelson (2019) show in their article, "Collaboration After Disaster: Explaining Intergovernmental Collaboration During the EPA Gold King Mine and TVA Coal Ash Recoveries," that problem complexity, unresolved historical issues and action type are likely to impede intergovernmental collaboration during recovery. Particularly longer term collaborative action, such as related to site remediation, seemed to invite conflict with more frequency and intensity.

The article by Wang (2019), "Disaster Response After Extreme Events in Taiwan" focuses on the intricacies of inter-organizational interaction in the more immediate response to extreme events. Wang looks into the role of formal institutions and their impact on the organization and performance in disaster management after two disasters in Taiwan: the ChiChi Earthquake in 1999 and Typhoon Morakot in 2009. The study concludes that formal institutions that had been lacking in the 1999 disaster response more clearly defined the boundaries and set qualifications that determined which organizations could legitimately participate in the disaster response in 2009. This gave some actors their necessary legal backing, but inhibited the activities of non-legitimated organizations, such as self-organized search and rescue communities. Also, formal institutions assigned specific roles and functions to disaster response organizations that formed interaction barriers between organizations that needed to cooperate across functional jurisdictions. The article ends with practical implications of its findings for disaster response policy.

Perhaps the odd one out in this RHCPP issue with respect to disasterrelatedness, but no less important in terms of topic and findings is the final article by Liu, Mumpower, Portney, and Vedlitz (2019): "Perceived Risk of Terrorism and Policy Preferences for Government Counterterrorism Spending." The authors show how individual social-economic-political characteristics and psychometric factors shape citizens' terrorism risk perception. The latter factors-perceived severity of the potential consequences of a terrorist attack, perceived number of 
persons affected, and perceived likelihood of an attack-have more influence on risk perception than social-economic-political traits. In turn, citizens' perception of risk and government competence positively affects their policy preference for government counterterrorism spending, also referred to as "willingness to pay" (WTP). The results, from two separate data waves of a US national panel survey, are heartening about the replicability of behavioral science research: they are both consistent with each other and with previous research. The study contributes not only to our understanding of the predictors of risk perception and willingness to pay but also to our understanding of change and particularly stability of those variables over time.

Sanneke Kuipers, editor-in-chief RHCPP.

\section{References}

Boin, Arjen, and Paul 't Hart. 2003. "Public Leadership in Times of Crisis: A Mission Impossible?" Public Administration Review 63 (5): 544-53.

Fisk, Jonathan, AJ Good, and Steven Nelson. 2019. “Collaboration After Disaster: Explaining Intergovernmental Collaboration During the EPA Gold King Mine and TVA Coal Ash Recoveries." Risk, Hazards and Crisis in Public Policy 10 (1): Xx-xx.

Jong, Wouter, and Michel Dückers. 2019. "The Perspective of the Affected: What People Confronted With Disasters Expect From Government Officials and Public Leaders." Risk, Hazards and Crisis in Public Policy 10 (1): Xx-xx.

Kuipers, Sanneke, and Nicholas Welsh. 2017. "Taxonomy of the Crisis and Disaster Literature: Themes and Types in 34 Years of Research." Risk, Hazards and Crisis in Public Policy 8 (4): 272-83.

La Porte, Todd R. 2018. "Preparing for Anomalies, Revealing the Invisible: Public Organization Puzzles." Risk, Hazards and Crisis in Public Policy 9 (3): 258-77.

Lai, Betty S., Ann-Margaret Esnard, Chris Wyczalkowski, Ryan Savage, and Hazel Shah. 2019. "Trajectories of School Recovery After a Natural Disaster: Risk and Protective Factors." Risk, Hazards and Crisis in Public Policy 10 (1): Xx-xx.

Liu, Xinsheng, Jeryl Mumpower, Kent Portney, and Arnold Vedlitz. 2019. “Perceived Risk of Terrorism and Policy Preferences for Government Counterterrorism Spending: Evidence From a U.S. National Panel Survey." Risk, Hazards and Crisis in Public Policy 10 (1): Xx-xx.

McCann, Erin. 2016. "Solving a Mystery Behind 'the Deadly Tsunami of Molasses' of 1919." The New York Times (November 28, 2016): p. A11.

Shanahan, Mark. 2019. “The Great Molasses Flood of 1919 was Boston's Strangest Disaster." The Boston Globe (January 9, 2019).

Wang, Wen-Jiun. 2019. “Disaster Response After Extreme Events in Taiwan: The Influence of Formal Institutions on Inter-Organizational Interaction." Risk, Hazards and Crisis in Public Policy 10 (1): Xx$\mathrm{xx}$.

\section{灾害后果与协作}

Sanneke Kuipers，公共政策之风险、灾害和危机 主编

一百年前, 正值一年之初, 一波高达 40 英尺的糖蜜以每小时 35 英里的速度席卷了 波士顿North End地区。逃难的人们无法超越糖蜜泄漏的速度，这次事件中 21 人死亡, 
受伤人数超过 150 。2 230 万加仑的糖蜜在喷出位于波士顿港口的储存罐的刹那，便形成 一股巨浪，所到之处皆被摧毁（McCann，2016）。当天纽约时报的一篇文章报道了这 一系列事件、死亡人数和造成的损害、以及房屋受到影响的所有者。这次事件突出

了两个特别主题：爆炸和随即喷出的糖蜜所破坏的临近消防站——消防紧急事件服务 区域被淹没一一结果则是，第一个前来施救的人员来自附近的航海学校，随后储备警 察和最近的军队驻点军人也很快赶来救援 (NYT, January 15，1919)。很显然，任何时 刻都有预料不到的运气和网络化的协作危机治理。

1919年纽约时报显然没有报道此次事件的潜在原因、责任或问责方面的问题、公 共卫生效果、亦或是受直接影响的公民最终结果如何。一方面, 这是因为针对此事的 哈佛模拟研究将会花费近100年的时间来最终确定伤亡人数 (Shanahan, 2019)。其他 危机研究点，例如问责、卫生风险和公民看法等也会花费相当长的时间来获取结果。

2019年第一期 公共政策之风险、灾害和危机 (RHCPP) 报道了关于公民的灾害 结果，和灾害响应协作中的复杂性。首先，作者Wouter Jong 和 Michel Dückers （2019）在 受影响人群的看法 一文中聚焦于危机发生后的心理社会支持。此文结 论认为, 受访者高度重视政府长期援助的公正、平等、同情和信赖。受影响人群还期 望 “在满足与危机事件相关的实际需求方面得到支持，并认为政府应该发挥能力动员 其网络伙伴，打破官僚障碍” (2019：p. XX)。由于公民所表达的明确灾后关爱期望在 危机和灾害文献中还是一个需要研究的话题，此文的实证发现可能为相关从业人员提 供宝贵的见解。作者肯定了由Boin 和' t t Hart (2003：547 - 548) 概述的期望差距，即 公共期望和公共领导者真正能在危机中实现的救助之间所存在的差异。

下一篇文章的作者分别是Betty Lai、Ann - Margaret Esnard、Chris Wyczalkowski、 Ryan Savage 和 Hazel Shah (2019), 他们检验了另一个需要研究的话题: 灾后的不同恢复模式。作者检验了和灾后学校恢复相关的风险和解释因素。作者发 现，低出勤率和高数量的经济贫困学生是与学校恢复相关的显著风险因素。作者强 调，其研究发现再次表明了灾害并不会平等地影响公民或社区，并且长期看来灾害影 响的差异会持续。因此，针对灾后恢复的政策提议应考虑 “低水平学校在灾后不良结 果一事上面临着最高风险” (Lai et a1, 2019, p. xx)。

公共机构间就灾后恢复工作展开的合作并不轻松。作者Jonathan Fisk、AJ Good 和 Steven Nelson在文章 灾后合作：阐述政府间合作一一以环境保护局金王矿泄漏 事件和田纳西河谷管理局煤灰泄漏事件后的恢复工作为例 中表明：问题复杂性、待 解决的历史问题和行动类型有可能妨碍灾后恢复期间的政府间合作。特别是较长期 的合作行动，例如与现场补救相关的行动，似乎会招致更多冲突。

作者Wen - Jiun Wang (2019) 所撰写的 台湾极端事件后的灾害响应 聚焦于政 府间互动的复杂性一一对极端事件采取的立即响应。作者考察了正式制度的作用和 其对台湾两次灾害事件后灾害管理的组织和表现产生的影响。这两次灾害分别为 1999年的集集地震和2009年的莫拉克龙卷风。本文结论认为，1999年灾害响应中所缺 少的正式制度更清晰地定义了界限, 并设定了相关资质以确定哪些组织能合法地参与 2009年灾害响应。这为一些行为者提供了必要的法律支持，但禁止了非法组织的相关 活动，例如自组织搜寻和救援社区。同样，正式制度为灾害响应组织分配了明确的角 色和功能, 这便为需要在不同管辖范围内进行合作的各个组织产生了互动障碍。文章 最后为灾害响应政策提供了实际意义。

或许本期RHCPP中较为不寻常地与灾害相关，但其话题和研究发现却同样重要的 文章便是最后这篇由作者Xinsheng Liu、Jeryl Mumpower、Kent Portney 和 Arnold Vedlitz撰写的 恐怖主义风险认知和政府反恐支出的政策偏好。作者展示了个人 的社会经济政治特征和心理因素如何塑造公民的恐怖主义风险认知。比起社会经济 
政治特征，之后的因素，即对恐怖袭击的潜在后果严重性的感知、对受影响人数的感 知, 和对袭击可能性的感知, 更能影响风险感知。反过来, 公民对风险和政府能力的 感知能积极影响其对政府反恐支出的政策偏好，这也被称之为 “支付意愿” (WTP)。 两波分开的美国国家调查数据结果赞赏了行为科学研究的可复制性：它们都相互保持 一致, 也与之前研究相一致。这篇文章不仅有助于理解风险感知的预测物和支付意 愿, 还有助于理解变化, 尤其是这些变量的稳定性随时间推移 (而产生的变化)。

\section{参考文献}

Boin, Arjen, and Paul 't Hart. 2003. "Public Leadership in Times of Crisis: A Mission Impossible?" Public Administration Review 63 (5): 544-53.

Fisk, Jonathan, AJ Good, and Steven Nelson. 2019. “Collaboration After Disaster: Explaining Intergovernmental Collaboration During the EPA Gold King Mine and TVA Coal Ash Recoveries." Risk, Hazards and Crisis in Public Policy 10 (1): Xx-xx.

Jong, Wouter, and Michel Dückers. 2019. "The Perspective of the Affected: What People Confronted With Disasters Expect From Government Officials and Public Leaders." Risk, Hazards and Crisis in Public Policy 10 (1): Xx-xx.

Kuipers, Sanneke, and Nicholas Welsh. 2017. “Taxonomy of the Crisis and Disaster Literature: Themes and Types in 34 Years of Research." Risk, Hazards and Crisis in Public Policy 8 (4): 272-83.

La Porte, Todd R. 2018. “Preparing for Anomalies, Revealing the Invisible: Public Organization Puzzles." Risk, Hazards and Crisis in Public Policy 9 (3): 258-77.

Lai, Betty S., Ann-Margaret Esnard, Chris Wyczalkowski, Ryan Savage, and Hazel Shah. 2019. "Trajectories of School Recovery After a Natural Disaster: Risk and Protective Factors." Risk, Hazards and Crisis in Public Policy 10 (1): Xx-xx.

Liu, Xinsheng, Jeryl Mumpower, Kent Portney, and Arnold Vedlitz. 2019. "Perceived Risk of Terrorism and Policy Preferences for Government Counterterrorism Spending: Evidence From a U.S. National Panel Survey." Risk, Hazards and Crisis in Public Policy 10 (1): Xx-xx.

McCann, Erin. 2016. "Solving a Mystery Behind 'the Deadly Tsunami of Molasses' of 1919." The New York Times (November 28, 2016): p. A11.

Shanahan, Mark. 2019. “The Great Molasses Flood of 1919 was Boston's Strangest Disaster." The Boston Globe (January 9, 2019).

Wang, Wen-Jiun. 2019. “Disaster Response After Extreme Events in Taiwan: The Influence of Formal Institutions on Inter-Organizational Interaction." Risk, Hazards and Crisis in Public Policy 10 (1): Xx$\mathrm{xx}$.

\section{Colaboración y consecuencias de los desastres}

\section{Sanneke Kuipers, editora principal RHCPP}

Hace un siglo, una ola de jarabe pegajoso de hasta 40 metros de altura inundó el área de North End de Boston a una velocidad de 35 millas por hora. No hubo forma de escapar de ola, que mató a 21 personas y dejó heridas a más de 150 . Una vez que los 2.3 galones de melaza se habían salido de un tanque de almacenamiento en el área de la Bahía de Boston, formaron una inundación que 
destruyó todo a su paso (McCann, 2016). Un artículo del New York Times en ese desafortunado día reporta acerca de los sucesos, las muertes y daños y acerca de los dueños de la propiedad en cuestión. Dos temas de interés particular sobresalen: la explosión y la inundación de melaza destruyeron la estación de bomberos adyacente-lo cual hizo que el área perdiera sus servicios de emergencia-así que los primeros en venir a socorrer fueron los oficiales de una escuela naval cercana, a quienes se les unieron las reservas de policía y ejército de la base militar más cercana (NYT, 15 de enero de 2019). Aparentemente la serendipia inesperada (La Porte, 2018: 260) y la gobernanza de crisis colaborativa en red (Kuipers y Welsh, 2017: 280) son de todas las épocas.

El New York Times de 1910 típicamente no reporta las causas principales, los problemas de rendición de cuentas o de responsabilidad, o los efectos en la salud pública para los ciudadanos afectados. Una de las razones es que pasarían casi cien años hasta que un estudio basado en un simulacro encontrara finalmente la causalidad (Shanahan, 2019). La mayoría de los otros intereses de la investigación de crisis, como la rendición de cuentas, las perspectivas de los ciudadanos y los riesgos de salud también necesitarían tiempo para ganar terreno.

La primera edición de Risk, Hazards and Crisis in Public Policy (RHCPP) de 2019 reporta las consecuencias de los desastres para los ciudadanos y las particularidades de la colaboración durante la respuesta a las crisis. Para empezar, Wouter Jong and Michel Dückers (2019) en su artículo, "La perspectiva de los afectados" se enfoca en el apoyo psicosocial después de las crisis. Concluyen que sus participantes valoraban altamente la justicia, la igualdad, la compasión y la confiabilidad de la ayuda del gobierno a largo plazo. Los ciudadanos afectados también esperaban "el apoyo del gobierno para satisfacer las necesidades prácticas relacionadas con los eventos, y asumieron que el gobierno usaría su capacidad para alinear a los socios de la red y romper las barreras burocráticas" (2019: p.XX). Dado que las expectativas explícitas de atención por parte de los ciudadanos frente a desastres son un tema poco investigado en la literatura sobre crisis y desastres, estos hallazgos empíricos pueden proporcionar información valiosa para los profesionales. Confirman las brechas en las expectativas descritas por Boin y t’h Hart (2003: 547-548), sobre la discrepancia entre las expectativas públicas y lo que los líderes públicos realmente pueden ofrecer en una crisis.

El siguiente artículo, de Betty Lai, Ann-Margaret Esnard, Chris Wyczalkowski, Ryan Savage y Hazel Shah (2019), examina otro tema poco estudiado: los diferentes patrones de recuperación después del desastre. Los autores analizan los riesgos y los factores explicativos relacionados con la recuperación de desastres en las escuelas. Ellos encuentran que la baja asistencia escolar y un alto número de estudiantes económicamente desfavorecidos son factores de riesgo significativos para la recuperación escolar. Los autores enfatizan que sus hallazgos muestran una vez más que los desastres no afectan a los ciudadanos o las comunidades por igual, y que la discriminación en el impacto persiste a largo plazo. Las iniciativas de políticas dirigidas a la recuperación deben tener en cuenta que "las escuelas con un rendimiento relativamente bajo tienen el 
mayor riesgo de resultados adversos después del desastre" (Lai et al, 2019, p. $\mathrm{Xx})$.

La cooperación posterior al desastre en los esfuerzos de recuperación entre instituciones públicas no es tarea fácil. Jonathan Fisk, AJ Good y Steven Nelson (2019) muestran en su artículo, "Colaboración después del desastre: Explicación de la colaboración intergubernamental durante la recuperación de la mina de oro de la EPA y de las cenizas de carbón de TVA", es probable que surjan problemas de complejidad, problemas históricos no resueltos y de tipo de acción que puedan impedir la colaboración intergubernamental durante la recuperación. Las acciones de colaboración a más largo plazo, como las relacionadas con la remediación del sitio, parecían invitar al conflicto con mayor frecuencia e intensidad.

El artículo de Wen-Jiun Wang (2019), "Respuesta ante desastres después de eventos extremos en Taiwán" se centra en las complejidades de la interacción entre organizaciones en la respuesta más inmediata a los eventos extremos. Wang analiza el papel de las instituciones formales y su impacto en la organización y el desempeño en la gestión de desastres después de dos desastres en Taiwán: el terremoto ChiChi en 1999 y el tifón Morakot en 2009. El estudio concluye que las instituciones formales que faltaban en el desastre de 1999 definieron más claramente los límites y establecieron las calificaciones que determinaron qué organizaciones podrían participar legítimamente en la respuesta a desastres en 2009. Esto brindó a algunos actores el respaldo legal necesario, pero inhibió las actividades de organizaciones no legitimadas, como la búsqueda y comunidades de rescate autoorganizadas. Además, las instituciones formales asignaron roles y funciones específicas a las organizaciones de respuesta a desastres que formaron barreras de interacción entre las organizaciones que necesitaban cooperar en las jurisdicciones funcionales. El artículo termina con las implicaciones prácticas de sus hallazgos para la política de respuesta ante desastres.

Quizás el texto extraño en esta edición de RHCPP con respecto al desastre, pero no menos importante en términos de tema y hallazgos, es el artículo final de Xinsheng Liu, Jeryl Mumpower, Kent Portney y Arnold Vedlitz: "Riesgo percibido de terrorismo y preferencias políticas para el gasto antiterrorismo del gobierno". Los autores muestran cómo las características socioeconómicas y políticas individuales y los factores psicométricos dan forma a la percepción del riesgo de terrorismo de los ciudadanos. Los últimos factores, la severidad percibida de las consecuencias potenciales de un ataque terrorista, la cantidad percibida de personas afectadas y la probabilidad percibida de un ataque, tienen más influencia en la percepción del riesgo que los rasgos socioeconómicos-políticos. A su vez, la percepción del riesgo y la competencia del gobierno de los ciudadanos afecta positivamente a su preferencia política por el gasto del contraterrorismo del gobierno, también conocida como "disposición a pagar" (WTP). Los resultados de una encuesta, a partir de dos grupos de datos separados de un panel nacional de EE. UU., Son alentadores acerca de la replicabilidad de la investigación de la ciencia del comportamiento: ambos son coherentes entre sí y con la investigación anterior. El estudio 
contribuye no solo a nuestra comprensión de los factores predictivos de la percepción del riesgo y la disposición a pagar, sino también a nuestra comprensión del cambio y, en particular, a la estabilidad de esas variables a lo largo del tiempo.

\section{Referencias}

Boin, Arjen, and Paul 't Hart. 2003. "Public Leadership in Times of Crisis: A Mission Impossible?" Public Administration Review 63 (5): 544-53.

Fisk, Jonathan, AJ Good, and Steven Nelson. 2019. “Collaboration After Disaster: Explaining Intergovernmental Collaboration During the EPA Gold King Mine and TVA Coal Ash Recoveries." Risk, Hazards and Crisis in Public Policy 10 (1): Xx-xx.

Jong, Wouter, and Michel Dückers. 2019. "The Perspective of the Affected: What People Confronted With Disasters Expect From Government Officials and Public Leaders." Risk, Hazards and Crisis in Public Policy 10 (1): Xx-xx.

Kuipers, Sanneke, and Nicholas Welsh. 2017. "Taxonomy of the Crisis and Disaster Literature: Themes and Types in 34 Years of Research." Risk, Hazards and Crisis in Public Policy 8 (4): 272-83.

La Porte, Todd R. 2018. "Preparing for Anomalies, Revealing the Invisible: Public Organization Puzzles." Risk, Hazards and Crisis in Public Policy 9 (3): 258-77.

Lai, Betty S., Ann-Margaret Esnard, Chris Wyczalkowski, Ryan Savage, and Hazel Shah. 2019. "Trajectories of School Recovery After a Natural Disaster: Risk and Protective Factors." Risk, Hazards and Crisis in Public Policy 10 (1): Xx-xx.

Liu, Xinsheng, Jeryl Mumpower, Kent Portney, and Arnold Vedlitz. 2019. “Perceived Risk of Terrorism and Policy Preferences for Government Counterterrorism Spending: Evidence From a U.S. National Panel Survey." Risk, Hazards and Crisis in Public Policy 10 (1): Xx-xx.

McCann, Erin. 2016. "Solving a Mystery Behind 'the Deadly Tsunami of Molasses' of 1919." The New York Times (November 28, 2016): p. A11.

Shanahan, Mark. 2019. "The Great Molasses Flood of 1919 was Boston's Strangest Disaster." The Boston Globe (January 9, 2019).

Wang, Wen-Jiun. 2019. “Disaster Response After Extreme Events in Taiwan: The Influence of Formal Institutions on Inter-Organizational Interaction." Risk, Hazards and Crisis in Public Policy 10 (1): Xx$\mathrm{xx}$. 